\title{
Desenvolvimento pós-embrionário de Ceraeochrysa cincta (Schneider, 1851) (Neuroptera: Chrysopidae) alimentada com ovos de Diatraea saccharallis (Fabricius, 1794) (Lepidoptera: Crambidae)
}

\author{
Post-embryonic development of Ceraeochrysa cincta (Schneider, \\ 1851) (Neuroptera: Chrysopidae) fed with eggs of Diatraea \\ saccharallis (Fabricius, 1794) (Lepidoptera: Crambidae)
}

\author{
Luis Gustavo Amorim Pessoa ${ }^{1 *}$; Sérgio de Freitas²; Elisângela de Souza Loureiro ${ }^{1}$
}

\section{Resumo}

Os crisopídeos são importantes predadores encontrados associados a diversos artrópodes-praga em diversos agroecossistemas. Para a criação massal desses predadores são utilizados tradicionalmente ovos de Sitotroga cerealella e de Anagasta kuehniella. Outros alimentos podem ser utilizados em substituição aos tradicionais, mas pouco se conhece sobre a biologia de crisopídeos e outros alimentos alternativos. Assim, este trabalho objetivou verificar o efeito de diferentes dietas sobre o desenvolvimento pós-embrionário dessa espécie em laboratório. Após a individualização em tubos de vidro, cada larva foi alimentada com ovos de $S$. cerealella e Diatraea saccharallis. Avaliou-se a duração, o peso e a viabilidade das larvas em cada ínstar, a duração o peso e viabilidade das fases de pré-pupa e pupa e a duração do período pós-embrionário. Adotou-se o delineamento inteiramente casualizado, com dez repetições por tratamento, cada uma constituída por cinco ovos ou larvas de C. cincta. As larvas foram pesadas 48 horas após a eclosão e 48 horas após cada ecdise, e as pré-pupas e pupas, 48 horas após iniciarem esses estágios Não houve interferência do tipo de alimento fornecido sobre os parâmetros avaliados. Desta forma, ovos de D. saccharallis podem ser utilizados para alimentação de C. cincta.

Palavras-chave: Crisopídeo, alimentação larval, controle biológico

\begin{abstract}
The lacewings (Ceraeochrysa cincta) are important predators found associated to many pests in several agroecosystems. Eggs of Sitotroga cerealella and Anagasta kuehniella are used traditionally for massal rearing of these predators. Other foods can be used in substitution to the traditional ones, but little is known about the biology of lacewings and other alternative foods. So, this work was carried out to evaluate the effect of different diets on the post-embryonic development of C. cincta in laboratory. After been individualized in glass tubes, each larva was fed with $S$. cereallella and Diatraea saccharallis. It was evaluated the larvae duration, weight and viability in each instar, the duration, weight and viability of the pupa and pre-pupa stages and the duration of the post-embryonic period. It was adopted a completely randomized design with ten replications per treatment, each consisting of five eggs or larvae of $C$. cincta. Larvae were weighed 48 hours after hatching and 48 hours after each molt, and pre-pupae and pupae, 48 hours after starting these stages. There was no effect of the kind of food provided on the parameters evaluated. Therefore, $D$. saccharallis eggs can be used to feed C. cincta.
\end{abstract}

Key words: Lacewing, larval feeding, biological control

\footnotetext{
${ }^{1}$ Docente da Universidade Federal de Mato Grosso do Sul, UFMS, Campus de Chapadão do Sul, CPCS, Rodovia MS 306 Estrada do Pasto Ruim, Caixa Postal 112, CEP 79560-000, Chapadão do Sul, MS. E-mail: luis.pessoa@ufms.br, lis_loureiro@ yahoo.com.br

${ }^{2}$ Docente da Universidade Estadual Paulista "Júlio de Mesquita Filho", UNESP Campus Jaboticabal. Departamento de Entomologia, Laboratório de Bioecologia de Crisopídeos. E-mail: serfre@fcav.unesp.br

* Autor para correspondência
} 
Os crisopídeos são importantes predadores encontrados associados a artrópodos-praga em vários agroecossistemas e que apresentam incidência estacional ou não e tegumento facilmente perfurável (FREITAS, 2001a).

Várias espécies são relatadas no Brasil. Dentre elas, Ceraeochrysa cincta (Schneider, 1851) (Neuroptera: Chrysopidae) ocorre em pomares de citrus, goiaba e plantios de seringueira, milho e algodão (FREITAS; PENNY, 2001), com potencial para controle de pragas associadas a esses cultivos.

De acordo com Freitas (2001b), o sucesso de um programa de controle biológico depende da disponibilização de agentes para liberações em extensas áreas, o que só é possível com sua multiplicação em larga escala.

Considera-se que em uma criação massal devem ser levados em consideração fatores extrínsecos e intrínsecos. Dentre os extrínsecos, estão técnicas para multiplicação dos insetos, materiais empregados, a manipulação das fases de desenvolvimento, o controle de qualidade e os custos de produção. Os intrínsecos estão relacionados à fisiologia do inseto como a adaptabilidade à dieta, potencial reprodutivo, fecundidade e fertilidade. Esses fatores são influenciados pelas exigências nutricionais dos insetos (CARVALHO; SOUZA, 2000).

Assim, a quantidade e qualidade do alimento consumido na fase larval de insetos predadores afetam taxa de crescimento, tempo de desenvolvimento, peso, sobrevivência, bem como influenciam fecundidade, longevidade, movimentação e capacidade de competição de adultos (PARRA, 1991). Canard e Principi (1984) relataram que a qualidade do alimento fornecido às larvas de crisopídeos tem considerável influência no tempo de desenvolvimento preimaginal, no aumento do peso corpóreo e na viabilidade.

Para criação massal de crisopídeos faz-se necessária a utilização de presas alternativas, como ovos de Sitotroga cerealella (Oliver, 1819) e de Anagasta kuehniella (Zeller, 1879) (Lepidoptera:
Gelechiidae). Outras presas podem ser utilizadas em substituição ao alimento tradicional, mas pouco se conhece sobre aspectos nutricionais relacionados à criação de crisopídeos com alimentos alternativos. A broca da cana de açúcar Diatraea saccharallis (Fabricius, 1794) (Lepidoptera: Crambidae) apresenta potencial para criação de crisopídeos (BIAGIONI; FREITAS, 2001; PESSOA et al., 2005; BORTOLI; MURATA, 2007), além ser facilmente multiplicada em laboratórios e biofábricas no Brasil. Assim, o trabalho teve por objetivo de verificar o efeito do fornecimento de ovos de Diatraea saccharallis (Fabricius, 1794) (Lepidoptera: Crambidae) no desenvolvimento pósembrionário C. cincta.

Adultos de crisopídeos C. cincta foram coletados em seringal, levados a laboratório onde, após triagem e identificação, adultos de foram acondicionados em gaiolas de cloreto de polivinila (PVC). A partir da criação-estoque, os crisopídeos foram multiplicados e criados em laboratório (FREITAS, 2001b), sendo utilizados insetos da terceira geração para os bioensaios.

Os bioensaios foram conduzidos em sala climatizada a $25 \pm 2{ }^{\circ} \mathrm{C}, 60 \pm 10 \%$ de UR e fotofase de 12 horas, utilizando-se ovos com até 24 horas de idade, os quais foram individualizados em tubos de vidro vedados com filme plástico. Após a eclosão, cada larva foi alimentada com ovos de $S$. cerealella e D. saccharallis, em quantidades que superam a capacidade diária de consumo $(0,0025 \mathrm{~g} / \mathrm{dia}$ e 0,0060g/dia, respectivamente) assegurando adequada quantidade de alimento (FREITAS, 2001b).

Adotou-se o delineamento inteiramente casualizado, com dez repetições por tratamento, cada uma constituída por cinco ovos ou larvas de $C$. cincta, sendo avaliados a duração, peso e viabilidade das larvas em cada ínstar, a duração, peso e viabilidade das fases de pré-pupa e pupa e a duração do período pós-embrionário. As larvas foram pesadas 48 horas após a eclosão e 48 horas após cada ecdise, e as pré-pupas e pupas, 48 horas após iniciarem esses estágios (PESSOA et al., 2005). 
Os dados referentes à viabilidade dos ínstares e das fases de larva, pré-pupa e pupa foram transformados para arsen $(\mathrm{x} / 100)^{1 / 2}$. Aqueles referentes à duração dos ínstares, fase larval, fases de pré-pupa e pupa, peso de larvas, de pré-pupas e pupas foram transformados para $(x+0,5)^{1 / 2}$. Efetuouse a análise de variância e o teste de agrupamento de médias de Tukey, a 5\% de probabilidade.
A alimentação de $C$. cincta com ovos de $D$. saccharalis não promoveu mudanças nos valores de durações, viabilidade e peso corpóreo dos estádios e estágios de desenvolvimento avaliados quando comparado ao alimento tradicional $S$. cereallela (Tabelas 1 e 2).

Tabela 1. Duração (dias) ( \pm EP) dos ínstares, fase larval, pré-pupa, pupa e ciclo biológico de C. cincta com diferentes alimentos.

\begin{tabular}{cccccccc}
\hline Tratamento & 1 Ínstar & 2o Ínstar & 3o Ínstar & Fase larval & Pré-pupa & Pupa & Ciclo biológico \\
\hline${ }^{1}$ Dieta 1 & $5,0 \pm 0,00$ & $4,1 \pm 0,03$ & $4,8 \pm 0,02$ & $14,1 \pm 0,01$ & $5,2 \pm 0,01$ & $10,1 \pm 0,01$ & $30,1 \pm 0,01$ \\
${ }^{2}$ Dieta 2 & $5,0 \pm 0,02$ & $3,9 \pm 0,03$ & $4,7 \pm 0,04$ & $14,1 \pm 0,02$ & $5,2 \pm 0,01$ & $10,3 \pm 0,01$ & $30,3 \pm 0,02$ \\
\hline CV (\%) & 2,12 & 5,42 & 5,23 & 1,88 & 2,01 & 1,55 & 1,17 \\
\hline
\end{tabular}

Não houve diferença significativa entre os tratamentos pelo teste $\mathrm{F}(\mathrm{P}<0,05)$.

${ }^{1}$ Ovos de Sitotroga cerealella.

${ }^{2}$ Ovos de Diatraea sacharallis.

De acordo com Canard e Principi (1984) e Parra (1991), a nutrição quantitativa e qualitativa, durante o estágio imaturo de desenvolvimento dos insetos, pode afetar vários parâmetros do seu ciclo biológico, sendo a duração, o peso e a sobrevivência do período imaturo os mais afetados para crisopídeos.

Diferentes dietas fornecidas às larvas de Chrysoperla defreitasi Brooks, 1994 (Neuroptera: Chrysopidae), alimentadas com ovos de $D$. saccharallis, afetaram apenas larvas de 3o ínstar, proporcionando aumento significativo na duração desse estádio em relação àquelas alimentadas com ovos de $S$. cereallela. O tratamento com ovos da broca-da-cana, comparado com o alimento tradicional também interferiu na viabilidade de larvas de 1 ínstar e da fase larval. Para a fase de pupa verificaram-se viabilidades acima de 90\%, para os dois alimentos (BIAGIONI; FREITAS, 2001).

Pessoa et al. (2005) observaram que apenas a duração do $1^{\circ}$ instar de larvas de Chrysoperla raimundoi Freitas e Penny (2001) (Neuroptera:
Chrysopidae), alimentadas com ovos de $D$. saccharallis apresentou duração significativamente maior em relação àquelas alimentadas com ovos de $S$. cereallela, não havendo interferência sobre as viabilidades e pesos corpóreos dos diferentes estádios e estágios avaliados.

De acordo com Kubo (1993) diminuição significativa na viabilidade da fase larval de Chrysoperla externa (Hagen, 1861) (Neuroptera: Chrysopidae), além de aumento significativo na duração dos ínstares e das fases larval e de pupa, foram observados para os insetos que foram alimentados com ovos da broca da cana-deaçúcar. Para Ceraeochrysa cubana (Hagen, 1861) (Neuroptera: Chrysopidae), observou-se uma redução significativa na viabilidade da fase de pupa, com o mesmo alimento. A duração do $2^{\circ}$ ínstar dessa espécie também foi afetada, apresentando um aumento significativo para larvas alimentadas com ovos da broca da cana-de-açúcar. Essa tendência de alongamento também foi verificada para as duas espécies de crisopídeos quando se avaliou a duração do ciclo biológico. 
Tabela 2. Viabilidade (\%) ( \pm EP) dos ínstares, da fase larval, das fases de pré-pupa e pupa e peso (mg) $( \pm$ EP) dos ínstares, de pré-pupas e pupas de $C$. cincta em função de diferentes regimes alimentares.

\begin{tabular}{|c|c|c|c|c|c|c|}
\hline \multicolumn{7}{|c|}{ Viabilidade } \\
\hline Tratamento & 10 Ínstar & $2^{\circ}$ Instar & 3ㅇ́nstar & Fase larval & Pré-pupa & Pupa \\
\hline${ }^{1}$ Dieta 1 & $99,6 \pm 3,56$ & $100,0 \pm 0,00$ & $99,6 \pm 3,56$ & $98,4 \pm 4,75$ & $98,6 \pm 4,46$ & $99,5 \pm 3,75$ \\
\hline${ }^{2}$ Dieta 2 & $90,32 \pm 7,67$ & $100,0 \pm 0,00$ & $100,0 \pm 0,00$ & $90,3 \pm 7,67$ & $97,9 \pm 5,36$ & $98,5 \pm 6,84$ \\
\hline CV (\%) & 23,90 & 0,00 & 9,04 & 26,08 & 16,91 & 18,42 \\
\hline \multicolumn{7}{|c|}{ Peso } \\
\hline Tratamento & 1o Ínstar & \multicolumn{2}{|c|}{ 2o Ínstar } & 3o Ínstar & Pré-pupa & Pupa \\
\hline${ }^{1}$ Dieta 1 & $0,2 \pm 0,02$ & \multirow{2}{*}{\multicolumn{2}{|c|}{$\begin{array}{l}1,9 \pm 0,10 \\
1,9 \pm 0,10\end{array}$}} & $7,0 \pm 0,25$ & $7,2 \pm 0,03$ & $7,0 \pm 0,03$ \\
\hline${ }^{2}$ Dieta 2 & $0,3 \pm 0,01$ & & & $7,4 \pm 0,42$ & $7,3 \pm 0,02$ & $7,1 \pm 0,02$ \\
\hline CV (\%) & 3,95 & $1,9 \pm 0,10$ & 7,29 & 7,31 & 2,80 & 2,97 \\
\hline
\end{tabular}

Não houve diferença significativa entre os tratamentos pelo teste $\mathrm{F}(\mathrm{P}<0,05)$.

${ }^{1}$ Ovos de Sitotroga cerealella.

${ }^{2}$ Ovos de Diatraea sacharallis.

Fornecendo ovos de $S$. cereallela e A. kuehniella e ovos de D. saccharallis a larvas de Chrysopa paraguaya Navás, 1924 (Neuroptera: Chrysopidae), Bortoli e Murata (2007) verificaram aumentos significativos apenas nas durações do 1ำ ínstar e na fase larval, não havendo interferência dos diferentes regimes alimentares sobre as viabilidades dos ínstares e das fases de desenvolvimento.

Embora outros autores tenham encontrado respostas nutricionais distintas em relação ao alimento oferecido a espécies de neurópteras, para C. cincta não se verificou esta tendência. De acordo com Albuquerque (2009), a composição química das presas das quais as larvas de crisopídeos se alimentam é semelhante, apresentando proteínas ou aminoácidos, lipídios, carboidratos, vitaminas, mineraiseoutros compostos. Porém, asconcentrações de cada constituinte dessas categorias principais e sua acessibilidade ao predador variam de espécie para espécie, evidenciando diferentes desempenhos para diferentes espécies de crisopídeos.

Desta forma, vislumbra-se, para $C$. cincta a possibilidade de criação em laboratório, utilizandose ovos da broca-da-cana. A adequada aceitação do predador, ao alimento oferecido, indica uma possível vantagem dessa espécie para uso no controle biológico.

\section{Referências}

ALBUQUERQUE, G. S. Crisopídeos (Neuroptera: Chrysopidae). In: PANIZZI, A .R.; PARRA, J. R. P. (Ed.). Bioecologia e nutrição de insetos: base para o manejo integrado de pragas. Brasília: Embrapa Informação Tecnológica. 2009. p. 969-1022.

BIAGIONI, A.; FREITAS, S. de. Efeito de diferentes dietas sobre o desenvolvimento pós-embrionário de Chrysoperla defreitasi Brooks (Neuroptera: Chrysopidae). Neotropical Entomology, Londrina, v. 30, n. 2, p. 333-336, 2001.

BORTOLI, S. A.; MURATA, A. T. Aspectos biológicos de Chrysopa paraguaya, Navás, 1920 (Neuroptera: Chrysopidae), em condições de laboratório. Boletin de Sanidad Vegetal Plagas, Logroño, v. 33, n. 1, p. 35-42, 2007.

CANARD, M.; PRINCIPI, M. M. Life histories and behavior. In: CANARD, M.; SÉMÉRIA, Y.; NEW, T. R. (Ed.). Biology of Chrysopidae. The Hague: W. Junk Publishes, 1984. p. 57-149.

CARVALHO, C. F.; SOUZA, B. Métodos de criação e produção de crisopídeos. In: BUENO, V. H. P. (Ed.). Controle biológico de pragas: produção massal e controle de qualidade. Lavras: UFLA, 2000. p. 91-109.

FREITAS, S de. Criação de crisopídeos (bicho lixeiro) em laboratório. Funep: Jaboticabal, 2001b. 20 p.

. O uso de crisopídeos no controle biológico de pragas. Funep: Jaboticabal, 2001a. 66 p. 
FREITAS, S. de; PENNY, N. D. The green lacewings (Neuroptera: Chrysopidae) of Brazilian agro-ecosystems. Proceedings of the California Academy Sciences, San Francisco, v. 50, n. 19, p. 245-395, 2001.

KUBO, R. K. Efeitos de diferentes presas no desenvolvimento de Chrysoperla externa (Hagen, 1861) e Ceraeochrysa cubana (Hagen, 1861) (Neuroptera: Chrysopidae). 1993. Dissertação (Mestrado em Entomologia) - Universidade Estadual Paulista. Faculdade de Ciências Agrárias e Veterinárias de Jaboticabal, Jaboticabal.
PARRA, J. R. P. Consumo e utilização de alimentos por insetos. In: PANIZZI, A. R.; PARRA, J. R. P. (Ed.). Ecologia nutricional de insetos e suas implicações no manejo de pragas. São Paulo: Manole, 1991. p. 9-57.

PESSOA, L. G. A.; FREITAS, S.; RODRIGUES, K. C.; GRADIM, S. Uso de ovos de Diatraea saccharallis (Lepidoptera: Gelechiidae) para alimentação de larvas de Chrysoperla raimundoi (Neuroptera: Chrysopidae). Acta Scientiraum Agronomy, Maringá, v. 27, n. 4, p. 651-654, 2005. 
